\title{
Substitutions of the NBS1 gene and clinicopathological characteristics of young breast cancer patients
}

\author{
Roberta Vadeikien $\dot{1}^{*}$, \\ Aistė Savukaityté, \\ Rasa Ugenskiené ${ }^{1}$, \\ Jurgita Gudaitiené², \\ Elona Juozaityte் $\dot{e}^{2}$ \\ ${ }^{1}$ Oncology Research Laboratory, \\ Oncology Institute, \\ Lithuanian University \\ of Health Sciences, Eiveniu St. 2, \\ Kaunas 50009, Lithuania \\ ${ }^{2}$ Department of Oncology \\ and Haematology, \\ Hospital of the Lithuanian University \\ of Health Sciences, Eiveniu St. 2, \\ Kaunas 50009, Lithuania
}

The purpose of this study was to determine characteristics potentially related to NBS1 mutations and polymorphisms in young $(\leq 50$ years of age) breast cancer patients. Blood from 80 breast cancer patients was collected. NBS1 mutations c.657_661del, p.R215W, p.I171 $\mathrm{V}$, and polymorphisms $c .8360 G>C, c .30537 G>C$ were genotyped by the PCR-RFLP method. Two-sided Chi-square test was used for univariate analysis and logistic regression analysis was used to evaluate the odds ratio. No carriers of the c.657_661 del, p. $R 215 \mathrm{~W}$ and p.I171 $\mathrm{V}$ mutations were found. NBS1 c.8360G $>C$ logistic regression analysis showed that $G C$ and $C C$ genotypes compared with GG genotype had decreased risk of low grade tumour, 2.885-fold ( $\mathrm{OR}=2.885$, 95\% CI 0.173-0.735, $P=0.005$ ) and 2.186 -fold ( $\mathrm{OR}=2.186,95 \%$ CI $0.188-0.888, P=0.024)$, respectively. 8360 CC genotype (OR $=3.034,95 \%$ CI 0.156-0.778, $P=0.010)$ significantly increased the chances of HER2 amplification compared to GG genotype. NBS1 8360 GC genotype had a higher risk for breast cancer progression $(\mathrm{OR}=1.673,95 \% \mathrm{CI}$ $0.233-0.915, P=0.027$ ). The homozygote 8360 CC carriers had approximately a six times higher risk for the disease progress $(\mathrm{OR}=5.946,95 \%$ CI $0.098-0.585, P=0.002)$. The prevalence of triple negative breast cancer type was significantly higher in individuals with NBS1 8360 CC genotype (OR $=2.186,95 \%$ CI $0.188-0.888, P=0.024)$. Regarding $c .30537 G>C$ polymorphism, none of the genotypes had a significant influence on pathological characteristics. NBS1 gene $c .8360 G>C$ polymorphism might be associated with breast cancer aggressiveness in young breast cancer patients.

Keywords: breast cancer, mutation, polymorphism, NBS1 gene

\footnotetext{
* Corresponding author. Email: roberta.vadeikiene@lsmuni.lt; r.jankauskaite@gmail.com
} 


\section{INTRODUCTION}

It is estimated that breast cancer (BC) comprises nearly $25 \%$ of all cancers in women and it is one of major causes of women cancer mortality worldwide (Ferlay et al., 2012). Preventive breast cancer prophylactic, new diagnostics, and treatment options have improved survival of breast cancer but there is still a need of better understanding of the disease. Realisation of molecular breast cancer mechanisms established on the basis of genetic differences in patients is important to individual medicine, therapy optimization, and cancer prognosis. Recent studies have focused on genetic factors, which increase cancer risk, but there is little data about the association of the genetic variation with prognostic aspects of the malign disease (Lima et al., 2019).

Nibrin $(95 \mathrm{kDa})$, the expression product of the Nijmegen Breakage Syndrome 1 (NBS1) gene [OMIM 602667], - is responsible for maintaining genome stability. Hypomorphic mutations that lead to decreased functions of the NBS1 gene are responsible for the $\mathrm{Ni}$ jmegen Breakage Syndrome (NBS), a rare autosomal recessive syndrome characterized by microcephaly, growth retardation, immunodeficiency, and predisposition to different malignancies. Mostly lymphoid and haematological cancers are observed, however, an increased susceptibility to solid tumours for homozygotes or heterozygotes of the mutated NBS1 was reported (Cybulski et al., 2004; Steffen et al., 2004). Recently published data has provided the information that the mutated NBS1 gene has influence on breast cancer risk mainly in Slavic populations (Cybulski et al., 2019; Rusak et al., 2019). The highest rate of heterozygous carriers of present gene mutations is noted in Eastern and Central Europe, especially in Poland, the Czech Republic and Ukraine (Huzarski et al., 2013).

It is estimated that approximately $0.5 \%$ of the Eastern European population are heterozygous for 5 bp deletion 657_661 delACAAA (c.657_661del) in NBS1 (Varon et al., 2000). This truncating germ line mutation that re- side in 6th NBS1 gene exon leads to formation of two alternative forms of nibrin with a lower molecular weight, of approximately 26 and $70 \mathrm{kDa}$ (di Masi, Antoccia, 2008). Therefore majority of studies in this restricted European region analysed the NBS1 c.657_661del mutation association with breast cancer predisposition. Although an elevated risk of breast cancer has been observed among carriers of c.657_661del, it was not confirmed to be associated with the course of the disease. According to $c$.657_661del mutation status, Huzarski et al. (2013) identified that mutation carriers and non-carriers presented with similar clinicopathological breast tumour characteristics and survival rate.

Since the identification of the NBS1 gene, many other mutations have been discovered. Recently, missense substitutions $p . R 215 W$ and p.I171 $\mathrm{V}$ have been suggested to be breast cancer susceptibility factors (Cybulski et al., 2004; Bogdanova et al., 2008a; Roznowki et al., 2008). In the study by Roznowski et al. (2008), the association between clinicopathological tumour features and mutation status was analysed. Although no statistically significant association was reported, it was suggested that p.I171V mutation could be a negative prognostic factor.

It has been suggested that NBS1 polymorphisms contribute to the aetiology of malignancies. One of the most commonly studied NBS1 polymorphism is c.8360G $>C$ (rs1805794), which has been determined to increase the risk of specific cancer in relation to person's ethnicity ( $\mathrm{Lu}$ et al., 2009; He et al., 2014). Lu et al. (2006) findings suggest that NBS1 c.8360G $>C$ polymorphism and haplotypes may contribute to the aetiology of sporadic breast cancer in young non-Hispanic white woman. According to Sun et al. (2007), $C C$ genotype of this polymorphism is associated with increased neutropenic fever in breast cancer patients. Also, these studies assessed the contribution of $c .30537 G>C$ ( $r$ 1805787) polymorphism to increased breast cancer risk, but the results were inconclusive ( $\mathrm{Lu}$ et al., 2006; Sun et al., 2007). 
In the present study, we performed the analysis for NBS1 gene mutations (c.657_661del, p.I171V, p.R215W) and polymorphisms (c.8369G $>C, c .30537 G>C$ ) for young women with breast cancer diagnosis. The majority of the studies analyse the relative risk of breast cancer in the case of current gene substitutions (Lu et al., 2006; Steffen et al., 2006; Bogdanova et al., 2008b; di Masi, Antoccia, 2008; Wang et al., 2010). However, the aim of this research was to determine the possible association between NBS1 and clinicopathological features together with $\mathrm{BC}$ progression.

\section{MATERIALS AND METHODS}

\section{Study population}

Biological material was obtained from 80 breast cancer patients. In genetic analysis, young premenopausal women ( $\leq 50$ years of age) with I or II stage breast cancer were involved. All samples were analysed at the Oncology Research Laboratory of the Oncology Institute of the Lithuanian University of Health Sciences. The study was approved by the local Biomedical Research Ethical Committee (protocol number BE-2-13). Patients provided written informed consent for the participation in the study.

The data on tumour clinicopathological characteristics was obtained from medical records.

Tumour oestrogen, progesterone receptors, and HER2 (human epidermal growth factor receptor 2) status were determined with immunohistochemistry. In the case of HER2, it was considered positive when immunohistochemically detected HER2 $(3+)$ or $(2+)$ amplification was confirmed by positive Fluorescence in situ hybridization. Testing for HER2 status gave as a score of 0 to $3+$ that measured the amount of HER2 proteins on the surface of the cells from breast tumour biopsy. Score from 0 to $1+$ was considered as HER2-negative. A score of $2+$ and $3+$ was considered HER2-positive.

The majority of breast tumours were not larger than $2 \mathrm{~cm}(63.8 \%)$. Most of selected young breast cancer patients (85\%) had negative tumour stromal lymphocyte infiltration. Approximately half of the cases (48.8\%) were positive for lymph node involvement. The majority of the tumours $(68.8 \%)$ were well to moderate differentiated (grade 1 (G1) or grade $2(\mathrm{G} 2)$ ). Low grade (G1) is well differentiated breast cancer. Cancer cells look like normal breast cells and grow (spread) slowly. Intermediate grade (G2) is moderately differentiated breast cancer. Cancer cells look slightly different to normal breast cells. High grade (G3) is poorly differentiated breast cancer. Cancer cells look very different to normal breast cells. In the case of tumour receptor expression, almost half patients were positive for estrogen (51.2\%) and progesterone (46.3\%) receptors, HER2 amplification was determined in $22.5 \%$ of tumours. Most of breast cancer patients had Luminal A tumour subtype (47.5\%). Luminal B, HER2 and triple negative (tumours that does not have any of the receptors that are found in breast cancer, e.g. oestrogen, progesterone receptors, and HER2) breast cancer subtypes were determined in $12.5 \%, 10.0 \%$, and $30.0 \%$ of selected cases, respectively. During the follow-up period (of at least two years), $27.5 \%$ patients were documented with a local or systemic disease progression.

\section{Genetic analysis}

DNA was isolated from peripheral blood leukocytes using commercially available GeneJET genomic DNA purification kit, according to manufacturer's recommendations (Thermo Fisher Scientific Baltics, Lithuania). Polymerase chain reaction (PCR) primer sequences and polymerase chain reaction-restriction fragment length polymorphism (PCR-RFLP) conditions for genotyping are listed in Table 1. All restriction enzymes for PCR-RFLP were from Thermo Fisher Scientific Baltics, Lithuania. Genetic testing for current gene mutation or polymorphism was done as described above, with some modifications when necessary (Resnick et al., 2003; Lu et al., 2006; Bogdanova et al., 2008a; Bogdanova et al., 2008b). 
Table 1. Primer sequences, PCR-RFLP conditions for the analysis of NBS1 mutations and polymorphisms

\begin{tabular}{|c|c|c|c|c|c|}
\hline Substitution & Primer sequence & $\begin{array}{l}\text { Annealing tem- } \\
\text { perature }\left({ }^{\circ} \mathrm{C}\right)\end{array}$ & $\begin{array}{c}\text { Restriction } \\
\text { enzyme }\end{array}$ & $\begin{array}{c}\text { DNA fragment } \\
\text { size (bp) }\end{array}$ & Reference \\
\hline $\begin{array}{c}\text { NBS1 } \\
\text { c.657_661del }\end{array}$ & $\begin{array}{c}\text { F: 5’-ACCCACCTCTT- } \\
\text { GATGAACCA-3' } \\
\text { R: 5'-CTGTTTG- } \\
\text { GCATTCAAAAA-3' }\end{array}$ & 55 & - & $\begin{array}{c}\text { WT 112bp } \\
\text { Mutant 107bp }\end{array}$ & $\begin{array}{c}\text { Res- } \\
\text { nick et al., } \\
2003\end{array}$ \\
\hline $\begin{array}{c}N B S 1 \\
\text { p.R215W }\end{array}$ & $\begin{array}{c}\text { F: 5'-GGAAGTA- } \\
\text { AAAATGTTGATCT- } \\
\text { GTCAGTA-3' } \\
\text { R: 5'-TGAAATACGT- } \\
\text { TAACAACTACTG-3' }\end{array}$ & 60 & RsaI & $\begin{array}{l}\text { WT } 153+25 b p \\
\text { Mutant } 178 b p\end{array}$ & $\begin{array}{l}\text { Bogda- } \\
\text { nova et al., } \\
2008 \mathrm{a}\end{array}$ \\
\hline $\begin{array}{c}\text { NBS1 } \\
\text { p.I171V }\end{array}$ & $\begin{array}{c}\text { F: 5'-TCCT- } \\
\text { GAAAGCAGTT- } \\
\text { GAGTCC-3' } \\
\text { R: 5'-ACAAGCAT- } \\
\text { TAAAGAGGGAGT- } \\
\text { TAAC-3' }\end{array}$ & 59 & Tsp509I & $\begin{array}{l}\text { WT } 127+43 \mathrm{bp} \\
\text { Mutant } 170 \mathrm{bp}\end{array}$ & $\begin{array}{c}\text { Bogda- } \\
\text { nova et al., } \\
2008 b\end{array}$ \\
\hline $\begin{array}{c}N B S 1 \\
\text { c. } 8360 G>C\end{array}$ & $\begin{array}{c}\text { F: 5' - CGTCCAATTG- } \\
\text { TAAAGCCAGAA-3' } \\
\text { R: 5'-TCCTGAAA- } \\
\text { CAAGCATTA- } \\
\text { AAGAGG-3' }\end{array}$ & 54 & HinfI & $\begin{array}{c}G G \text { genotype } \\
\text { 125+49bp } \\
\text { CC genotype } \\
\text { 174bp } \\
\text { GC genotype } \\
174+125+49 \text { bp }\end{array}$ & $\begin{array}{l}\text { Lu et al., } \\
2006\end{array}$ \\
\hline $\begin{array}{c}N B S 1 \\
\text { c. } 30537 G>C\end{array}$ & $\begin{array}{c}\text { F: 5’-TTGCTTGAAT- } \\
\text { GACAGCCTGA-3' }\end{array}$ & 61 & EarI & $\begin{array}{c}\text { GG genotype } \\
197 \mathrm{bp} \\
\text { CC genotype } \\
172+25 \mathrm{bp} \\
\text { GC genotype } \\
197+172+25 \mathrm{bp}\end{array}$ & $\begin{array}{l}\text { Lu et al., } \\
2006\end{array}$ \\
\hline
\end{tabular}

* WT - wild type.

\section{Statistical analysis}

Statistical analysis was done with SPSS 20.0 for Windows (SPSS, Inc., Chicago, IL, USA). In the case of qualitative variables, comparison was made by the 2 -sided Pearson's Chi-square or 2-sided Monte Carlo exact test (in the cases where $>25 \%$ of the expected value were $<5$ ). In order to calculate the crude and adjusted odds ratio (OR) and their 95\% confidence intervals (CIs) (with and without adjustment for cancer diagnosis age), logistic regression analysis was used. Results were statistically significant at $P$ value lower than 0.05 .

\section{RESULTS}

Among the 80 patients, no carriers of the NBS1 c.657_661del, p.R215W, and p.I171V mutations were found. NBS1 c.8360G>C genotyping identified 11 patients with $G G(13.75 \%), 38$ with $G C$ (47.5\%), and 31 with CC (38.75\%). Regarding c. $30537 G>C$ polymorphism, 46 patients were $G G$ (57.5\%), 29 GC (36.25\%), and five with CC genotype (6.25\%). The genotypes of NBS1 c.8360G>C and $c .30537 G>C$ were under Hardy-Weinberg Equilibrium. The NBS1 polymorphism genotype distribution between pathological characteristics 
is summarized in Table 2. Our descriptive data ants do not statistically contribute to breast indicate that $c .8360 G>C$ and $c .30537 G>C$ vari-

cancer characteristics. Dominant and recessive

Table 2. The distribution of tumour clinicopathological characteristics by NBS1 genotype

\begin{tabular}{|c|c|c|c|c|c|c|c|c|}
\hline \multirow{4}{*}{ Variable } & \multicolumn{3}{|c|}{ NBS1 c.8360 } & \multirow{4}{*}{$\begin{array}{c}P \\
\text { value }\end{array}$} & \multicolumn{3}{|c|}{ NBS1 c.30537 } & \multirow{4}{*}{$\begin{array}{c}P \\
\text { value }\end{array}$} \\
\hline & \multirow{2}{*}{$\begin{array}{c}G G \\
\mathrm{~N}\end{array}$} & \multirow{2}{*}{$\begin{array}{c}G C \\
\mathrm{~N}\end{array}$} & \multirow{2}{*}{$\begin{array}{c}C C \\
\mathrm{~N}\end{array}$} & & \multirow{2}{*}{$\begin{array}{c}G G \\
\mathrm{~N}\end{array}$} & \multirow{2}{*}{$\frac{G C}{\mathrm{~N}}$} & \multirow{2}{*}{$\begin{array}{c}C C \\
\mathrm{~N}\end{array}$} & \\
\hline & & & & & & & & \\
\hline & $\%$ & $\%$ & $\%$ & & $\%$ & $\%$ & $\%$ & \\
\hline Tumour size & & & & 0.822 & & & & 0.118 \\
\hline \multirow{2}{*}{$<2 \mathrm{~cm}$} & 7 & 26 & 19 & & 34 & 16 & 2 & \\
\hline & 63.6 & 68.4 & 61.3 & & 73.9 & 55.2 & 40.0 & \\
\hline \multirow{2}{*}{$\geq 2 \mathrm{~cm}$} & 4 & 12 & 12 & & 12 & 13 & 3 & \\
\hline & 36.4 & 31.6 & 38.7 & & 26.1 & 44.8 & 60.0 & \\
\hline Histological grade & & & & 0.194 & & & & 0.403 \\
\hline \multirow{2}{*}{ Grade $1+2$} & 5 & 28 & 22 & & 34 & 17 & 4 & \\
\hline & 45.5 & 73.7 & 71.0 & & 73.9 & 58.6 & 80.0 & \\
\hline \multirow{2}{*}{ Grade 3} & 6 & 10 & 9 & & 12 & 12 & 1 & \\
\hline & 54.5 & 26.3 & 29.0 & & 26.1 & 41.4 & 20.0 & \\
\hline Stroma lymphocytic infiltration & & & & 0.843 & & & & 0.457 \\
\hline \multirow{2}{*}{ Positive } & 1 & 6 & 5 & & 6 & 6 & 0 & \\
\hline & 9.1 & 15.8 & 16.1 & & 13.0 & 20.7 & 0.0 & \\
\hline \multirow{2}{*}{ Negative } & 10 & 32 & 26 & & 40 & 23 & 5 & \\
\hline & 90.9 & $84.2 \%$ & 83.9 & & 87.0 & 79.3 & 100 & \\
\hline Lymph nodes & & & & 0.084 & & & & 0.549 \\
\hline \multirow{2}{*}{ Positive } & 8 & 20 & 11 & & 25 & 12 & 2 & \\
\hline & 72.7 & 52.6 & 35.5 & & 54.3 & 41.4 & 40.0 & \\
\hline \multirow{2}{*}{ Negative } & 3 & 18 & 20 & & 21 & 17 & 3 & \\
\hline & 27.3 & 47.4 & 64.5 & & 45.7 & 58.6 & 60.0 & \\
\hline Oestrogen receptor & & & & 0.670 & & & & 0.487 \\
\hline \multirow{2}{*}{ Positive } & 7 & 19 & 15 & & 26 & 12 & 3 & \\
\hline & 63.6 & 50.0 & 48.4 & & 56.5 & 41.4 & 60.0 & \\
\hline \multirow{2}{*}{ Negative } & 4 & 19 & 16 & & 20 & 17 & 2 & \\
\hline & 36.4 & 50.0 & 51.6 & & 43.5 & 58.6 & 40.0 & \\
\hline Progesterone receptor & & & & 0.662 & & & & 0.418 \\
\hline \multirow{2}{*}{ Positive } & 4 & 17 & 16 & & 21 & 15 & 1 & \\
\hline & 36.4 & 44.7 & 51.6 & & 45.7 & 51.7 & 20.0 & \\
\hline \multirow{2}{*}{ Negative } & 7 & 21 & 15 & & 25 & 14 & 4 & \\
\hline & 63.6 & 55.3 & 48.4 & & 54.3 & 48.3 & 80.0 & \\
\hline HER2 amplification & & & & 0.704 & & & & 0.316 \\
\hline \multirow{2}{*}{ Amplified } & 3 & 7 & 8 & & 13 & 4 & 1 & \\
\hline & 27.3 & 18.4 & 25.8 & & 28.3 & 13.8 & 20.0 & \\
\hline \multirow{2}{*}{ Non-amplified } & 8 & 31 & 23 & & 33 & 25 & 4 & \\
\hline & 72.7 & 81.6 & 74.2 & & 71.7 & 86.2 & 80.0 & \\
\hline
\end{tabular}


Table 2. (continued)

\begin{tabular}{|c|c|c|c|c|c|c|c|c|}
\hline \multirow{4}{*}{ Variable } & \multicolumn{3}{|c|}{ NBS1 c.8360 } & \multirow{4}{*}{$\begin{array}{c}P \\
\text { value }\end{array}$} & \multicolumn{3}{|c|}{ NBS1 c.30537 } & \multirow{4}{*}{$\begin{array}{c}P \\
\text { value }\end{array}$} \\
\hline & \multirow{3}{*}{$\begin{array}{c}G G \\
\mathbf{N} \\
\% \\
\end{array}$} & \multirow{3}{*}{\begin{tabular}{c|}
$G C$ \\
$\mathrm{~N}$ \\
$\%$ \\
\end{tabular}} & \multirow{3}{*}{$\begin{array}{c}C C \\
\mathrm{~N} \\
\% \\
\end{array}$} & & \multirow{3}{*}{$\begin{array}{c}G G \\
\mathrm{~N} \\
\%\end{array}$} & \multirow{3}{*}{$\begin{array}{c}G C \\
\mathrm{~N} \\
\%\end{array}$} & \multirow{3}{*}{$\begin{array}{c}C C \\
\mathrm{~N} \\
\% \\
\end{array}$} & \\
\hline & & & & & & & & \\
\hline & & & & & & & & \\
\hline \multicolumn{9}{|c|}{ Molecular breast cancer type } \\
\hline Luminal A & & & & 0.866 & & & & 0.718 \\
\hline \multirow{2}{*}{ Positive } & 6 & 18 & 14 & & 20 & 15 & 3 & \\
\hline & 54.5 & 47.4 & 45.2 & & 43.5 & 51.7 & 60.0 & \\
\hline \multirow{2}{*}{ Negative } & 5 & 20 & 17 & & 26 & 14 & 2 & \\
\hline & 45.5 & 52.6 & 54.8 & & 56.5 & 48.3 & 40.0 & \\
\hline Luminal B & & & & 0.896 & & & & 0.314 \\
\hline \multirow{2}{*}{ Positive } & 2 & 4 & 4 & & 8 & 2 & 0 & \\
\hline & 18.2 & 10.5 & 12.9 & & 17.4 & 6.9 & 0.0 & \\
\hline \multirow{2}{*}{ Negative } & 9 & 34 & 27 & & 38 & 27 & 5 & \\
\hline & 81.8 & 89.5 & 87.1 & & 82.6 & 93.1 & 100.0 & \\
\hline HER2 type & & & & 0.876 & & & & 0.729 \\
\hline \multirow{2}{*}{ Positive } & 1 & 3 & 4 & & 5 & 2 & 1 & \\
\hline & 9.1 & 7.9 & 12.9 & & 10.9 & 6.9 & 20.0 & \\
\hline \multirow{2}{*}{ Negative } & 10 & 35 & 27 & & 41 & 27 & 4 & \\
\hline & 90.9 & 92.1 & 87.1 & & 89.1 & 93.1 & 80.0 & \\
\hline Triple negative & & & & 0.587 & & & & 0.737 \\
\hline \multirow{2}{*}{ Positive } & 2 & 13 & 9 & & 13 & 10 & 1 & \\
\hline & 18.2 & 34.2 & 29.0 & & 28.3 & 34.5 & 20.0 & \\
\hline \multirow{2}{*}{ Negative } & 9 & 25 & 22 & & 33 & 19 & 4 & \\
\hline & 81.8 & 65.8 & 71.0 & & 71.7 & 65.5 & 80.0 & \\
\hline Disease progress & & & & 0.410 & & & & 0.238 \\
\hline \multirow{2}{*}{ Present } & 4 & 12 & 6 & & 16 & 5 & 1 & \\
\hline & 36.4 & 31.6 & 19.4 & & 34.8 & 17.2 & 20.0 & \\
\hline \multirow{2}{*}{ Absent } & 7 & 26 & 25 & & 30 & 24 & 4 & \\
\hline & 63.6 & 68.4 & 80.6 & & 65.2 & 82.8 & 80.0 & \\
\hline
\end{tabular}

models showed no significant associations with studied characteristics in our study (Table 3 ).

The association between NBS1 polymorphisms and clinicopathological characteristics was also assessed by the odds ratio with its corresponding 95\% confidence interval. The logistic regression analysis showed no significant differences between NBS1 polymorphism genotype and pathological features when the model was adjusted for the diagnosis age. However, there was a significant association between $c .8360 G>C$ polymorphism and clinicopathological charac- teristics in the model without adjustment for the age of breast cancer diagnosis (Table 4). It was determined that heterozygous carriers of NBS1 8360 GC genotype had a 2.885-fold decreased risk of low grade (well-differentiated) $(\mathrm{OR}=2.885,95 \%$ CI $0.173-0.735, P=0.005)$ tumour compared with those with $G G$ genotype. The homozygote $C C$ genotype of this polymorphism carriers had a 2.186-fold $(\mathrm{OR}=2.186$, 95\% CI $0.188-0.888, P=0.024)$ decreased risk of low grade $(\mathrm{G} 1+2)$ tumour differentiation. 8360 CC genotype $(\mathrm{OR}=3.034,95 \% \mathrm{CI}$ 
Table 3. NBS1 polymorphisms genotypes models association with clinicopathological breast cancer characteristics

\begin{tabular}{|c|c|c|c|c|c|c|c|c|c|c|c|c|}
\hline \multirow{6}{*}{ Variable } & \multirow{3}{*}{\multicolumn{2}{|c|}{$\begin{array}{c}\text { NBS1c.8360 } \\
\text { Recessive } \\
\text { model }^{*} \\
\end{array}$}} & \multirow{6}{*}{$\begin{array}{c}P \\
\text { value }\end{array}$} & \multirow{3}{*}{\multicolumn{2}{|c|}{$\begin{array}{c}\text { NBS1c.8360 } \\
\text { Dominant } \\
\text { model }^{*} \\
\end{array}$}} & \multirow{6}{*}{$\begin{array}{c}P \\
\text { value }\end{array}$} & \multirow{3}{*}{\multicolumn{2}{|c|}{$\begin{array}{c}\text { NBS1 c.30537 } \\
\text { Recessive } \\
\text { model }^{*} \\
\end{array}$}} & \multirow{6}{*}{$\begin{array}{c}P \\
\text { value }\end{array}$} & \multirow{3}{*}{\multicolumn{2}{|c|}{$\begin{array}{c}\text { NBS1 c.30537 } \\
\text { Dominant } \\
\text { model }^{*} \\
\end{array}$}} & \multirow{6}{*}{$\begin{array}{c}P \\
\text { value }\end{array}$} \\
\hline & & & & & & & & & & & & \\
\hline & & & & & & & & & & & & \\
\hline & $\mathrm{GG}+\mathrm{GC}$ & $\mathrm{CC}$ & & GG & $\mathrm{GC}+\mathrm{CC}$ & & $\mathrm{GG}+\mathrm{GC}$ & $\mathrm{CC}$ & & GG & $\mathrm{GC}+\mathrm{CC}$ & \\
\hline & $\mathbf{N}$ & $\mathbf{N}$ & & $\mathbf{N}$ & $\mathbf{N}$ & & $\mathbf{N}$ & $\mathbf{N}$ & & $\mathbf{N}$ & $\mathbf{N}$ & \\
\hline & $\%$ & $\%$ & & $\%$ & $\%$ & & $\%$ & $\%$ & & $\%$ & $\%$ & \\
\hline \multicolumn{3}{|l|}{ Tumour size } & \multicolumn{3}{|l|}{0.468} & \multicolumn{3}{|l|}{0.727} & \multicolumn{3}{|l|}{0.337} & 0.052 \\
\hline \multirow{2}{*}{$<2 \mathrm{~cm}$} & 34 & 18 & & 9 & 43 & & 50 & 2 & & 18 & 34 & \\
\hline & 68.0 & 60.0 & & 69.2 & 64.2 & & 66.7 & 40.0 & & 52.9 & 73.9 & \\
\hline \multirow{2}{*}{$\geq 2 \mathrm{~cm}$} & 16 & 12 & & 4 & 24 & & 25 & 3 & & 16 & 12 & \\
\hline & 32.0 & 40.0 & & 30.8 & 35.8 & & 33.3 & 60.0 & & 47.1 & 26.1 & \\
\hline $\begin{array}{c}\text { Histological } \\
\text { grade }\end{array}$ & & & 0.852 & & & 0.205 & & & 0.673 & & & 0.247 \\
\hline \multirow{2}{*}{ Grade $1+2$} & 34 & 21 & & 6 & 48 & & 51 & 4 & & 21 & 34 & \\
\hline & 68.0 & 70.0 & & 46.2 & 71.6 & & 68.0 & 80.0 & & 61.8 & 73.9 & \\
\hline \multirow{2}{*}{ Grade 3} & 16 & 9 & & 7 & 19 & & 24 & 1 & & 13 & 12 & \\
\hline & 32.0 & 30.0 & & 53.8 & 28.4 & & 32.0 & 20.0 & & 38.2 & 26.1 & \\
\hline $\begin{array}{c}\text { Stroma } \\
\text { lymphocytic } \\
\text { infiltration }\end{array}$ & & & 0.746 & & & 0.420 & & & 0.593 & & & 0.569 \\
\hline \multirow{2}{*}{ Positive } & 7 & 5 & & 1 & 11 & & 12 & 0 & & 6 & 6 & \\
\hline & 14.0 & 16.7 & & 7.7 & 16.4 & & 16.0 & 0.0 & & 17.6 & 13.0 & \\
\hline \multirow{2}{*}{ Negative } & 43 & 25 & & 12 & 56 & & 63 & 5 & & 28 & 40 & \\
\hline & 86.0 & 83.3 & & 92.3 & 83.6 & & 84.0 & 100 & & 82.4 & 87.0 & \\
\hline $\begin{array}{l}\text { Lymph } \\
\text { nodes }\end{array}$ & & & 0.094 & & & 0.108 & & & $>0.999$ & & & 0.224 \\
\hline \multirow{2}{*}{ Positive } & 28 & 11 & & 9 & 30 & & 37 & 2 & & 14 & 25 & \\
\hline & 56.0 & 36.7 & & 69.2 & 44.8 & & 49.3 & 40.0 & & 41.2 & 54.3 & \\
\hline \multirow{2}{*}{ Negative } & 22 & 19 & & 4 & 37 & & 38 & 3 & & 20 & 21 & \\
\hline & 44.0 & 63.3 & & 30.8 & 55.2 & & 50.7 & 60.0 & & 58.8 & 45.7 & \\
\hline $\begin{array}{c}\text { Oestrogen } \\
\text { receptor }\end{array}$ & & & 0.525 & & & 0.156 & & & $>0.999$ & & & 0.273 \\
\hline Doritive & 27 & 14 & & 9 & 32 & & 38 & 3 & & 15 & 26 & \\
\hline Positive & 54.0 & 46.7 & & 69.2 & 47.8 & & 50.7 & 60.0 & & 44.1 & 56.5 & \\
\hline Nerntive & 23 & 16 & & 4 & 35 & & 37 & 2 & & 19 & 20 & \\
\hline Negative & 46.0 & 53.3 & & 30.8 & 52.2 & & 49.3 & 40.0 & & $55.9 \%$ & 43.5 & \\
\hline $\begin{array}{c}\text { Progester- } \\
\text { one receptor }\end{array}$ & & & 0.602 & & & 0.221 & & & 0.366 & & & 0.901 \\
\hline & 22 & 15 & & 4 & 33 & & 36 & 1 & & 16 & 21 & \\
\hline Positive & 44.0 & 50.0 & & 30.8 & 49.3 & & 48.0 & 20.0 & & 47.1 & 45.7 & \\
\hline Negative & 28 & 15 & & 9 & 34 & & 39 & 4 & & 18 & 25 & \\
\hline Negative & 56.0 & 50.0 & & 69.2 & 50.7 & & 52.0 & 80.0 & & 52.9 & 54.3 & \\
\hline $\begin{array}{l}\text { HER2 am- } \\
\text { plification }\end{array}$ & & & 0.890 & & & 0.957 & & & $>0.999$ & & & 0.151 \\
\hline
\end{tabular}


Table 3. (continued)

\begin{tabular}{|c|c|c|c|c|c|c|c|c|c|c|c|c|}
\hline \multirow{6}{*}{ Variable } & \multirow{3}{*}{\multicolumn{2}{|c|}{$\begin{array}{c}\text { NBS1c.8360 } \\
\text { Recessive } \\
\text { model }^{*}\end{array}$}} & \multirow{6}{*}{$\begin{array}{c}P \\
\text { value }\end{array}$} & \multirow{3}{*}{\multicolumn{2}{|c|}{$\begin{array}{c}\text { NBS1c.8360 } \\
\text { Dominant } \\
\text { model }^{*}\end{array}$}} & \multirow{6}{*}{$\begin{array}{c}P \\
\text { value }\end{array}$} & \multirow{3}{*}{\multicolumn{2}{|c|}{$\begin{array}{c}\text { NBS1 c.30537 } \\
\text { Recessive } \\
\text { model }^{*} \\
\end{array}$}} & \multirow{6}{*}{$\begin{array}{c}P \\
\text { value }\end{array}$} & \multirow{3}{*}{\multicolumn{2}{|c|}{$\begin{array}{c}\text { NBS1 c.30537 } \\
\text { Dominant } \\
\text { model }^{*}\end{array}$}} & \multirow{6}{*}{$\begin{array}{c}P \\
\text { value }\end{array}$} \\
\hline & & & & & & & & & & & & \\
\hline & & & & & & & & & & & & \\
\hline & $\mathrm{GG}+\mathrm{GC}$ & $\mathrm{CC}$ & & GG & $\mathrm{GC}+\mathrm{CC}$ & & GG+GC & $\mathrm{CC}$ & & GG & $\mathrm{GC}+\mathrm{CC}$ & \\
\hline & $\mathbf{N}$ & $\mathbf{N}$ & & $\mathbf{N}$ & $\mathbf{N}$ & & $\mathbf{N}$ & $\mathbf{N}$ & & $\mathbf{N}$ & $\mathbf{N}$ & \\
\hline & $\%$ & $\%$ & & $\%$ & $\%$ & & $\%$ & $\%$ & & $\%$ & $\%$ & \\
\hline \multirow{2}{*}{ Amplified } & 11 & 7 & & 3 & 15 & & 17 & 1 & & 5 & 13 & \\
\hline & 22.0 & 23.3 & & 23.1 & 22.4 & & $22.7 \%$ & 20.0 & & 14.7 & 28.3 & \\
\hline \multirow{2}{*}{$\begin{array}{l}\text { Non-ampli- } \\
\text { fied }\end{array}$} & 39 & 23 & & 10 & 52 & & 58 & 4 & & 29 & 33 & \\
\hline & 78.0 & 76.7 & & 76.9 & 77.6 & & $77.3 \%$ & 80.0 & & 85.3 & 71.7 & \\
\hline \multicolumn{13}{|l|}{$\begin{array}{c}\text { Molecular } \\
\text { breast can- } \\
\text { cer type }\end{array}$} \\
\hline Luminal A & & & 0.908 & & & 0.268 & & & 0.664 & & & 0.402 \\
\hline \multirow{2}{*}{ Positive } & 24 & 14 & & 8 & 30 & & 35 & 3 & & 18 & 20 & \\
\hline & 48.0 & 46.7 & & 61.5 & 44.8 & & 46.7 & 60.0 & & 52.9 & 43.5 & \\
\hline \multirow{2}{*}{ Negative } & 26 & 16 & & 5 & 37 & & 40 & 2 & & 16 & 26 & \\
\hline & 52.0 & 53.3 & & 38.5 & 55.2 & & 53.3 & 40.0 & & 47.1 & 56.5 & \\
\hline Luminal B & & & 0.600 & & & 0.731 & & & 0.619 & & & 0.124 \\
\hline \multirow{2}{*}{ Positive } & 7 & 3 & & 2 & 8 & & 10 & 0 & & 2 & 8 & \\
\hline & 14.0 & 10.0 & & 15.4 & 11.9 & & 13.3 & 0.0 & & 5.9 & 17.4 & \\
\hline \multirow{2}{*}{ Negative } & 43 & 27 & & 11 & 59 & & 65 & 5 & & 32 & 38 & \\
\hline & 86.0 & 90.0 & & 84.6 & 88.1 & & 86.7 & 100 & & 94.1 & 82.6 & \\
\hline HER2 type & & & 0.441 & & & 0.762 & & & $>0.999$ & & & $>0.999$ \\
\hline \multirow{2}{*}{ Positive } & 4 & 4 & & 1 & 7 & & 7 & 1 & & 3 & 5 & \\
\hline & 8.0 & 13.3 & & 7.7 & 10.4 & & 9.3 & 20.0 & & 8.8 & 10.9 & \\
\hline \multirow{2}{*}{ Negative } & 46 & 26 & & 12 & 60 & & 68 & 4 & & 31 & 41 & \\
\hline & 92.0 & 86.7 & & 92.3 & 89.6 & & 90.7 & 80.0 & & 91.2 & 89.1 & \\
\hline $\begin{array}{l}\text { Triple nega- } \\
\text { tive }\end{array}$ & & & $>0.999$ & & & 0.209 & & & $>0.999$ & & & 0.693 \\
\hline \multirow{2}{*}{ Positive } & 15 & 9 & & 2 & 22 & & 23 & 1 & & 11 & 13 & \\
\hline & 30.0 & 30.0 & & 15.4 & 32.8 & & 30.7 & 20.0 & & 32.4 & 28.3 & \\
\hline \multirow{2}{*}{ Negative } & 35 & 21 & & 11 & 45 & & 52 & 4 & & 23 & 33 & \\
\hline & 70.0 & 70.0 & & 84.6 & 67.2 & & 69.3 & 80.0 & & 67.6 & 71.7 & \\
\hline $\begin{array}{c}\text { Disease } \\
\text { progress }\end{array}$ & & & 0.245 & & & 0.478 & & & $>0.999$ & & & 0.090 \\
\hline \multirow{2}{*}{ Present } & 16 & 6 & & 5 & 17 & & 21 & 1 & & 6 & 16 & \\
\hline & 32.0 & 20.0 & & 38.5 & 24.4 & & 28.0 & 20.0 & & 17.6 & 34.8 & \\
\hline \multirow{2}{*}{ Absent } & 34 & 24 & & 8 & 50 & & 54 & 4 & & 28 & 30 & \\
\hline & 68.0 & 80.0 & & 61.5 & 74.6 & & 72.0 & 80.0 & & 82.4 & 65.2 & \\
\hline
\end{tabular}

* Dominance of the alleles can be assumed by combining the heterozygote and one of the homozygotes as a single group. In c.8360G >C polymorphism (same with c.30537G>C variant), $\mathrm{C}$ is the "risk" allele. In the recessive model, two copies of allele $\mathrm{C}$ required to affect breast cancer prognosis, i.e., $\mathrm{GG}+\mathrm{GC}$ vs $\mathrm{CC}$ are used in the analysis. The dominant effect of the "risk" C allele is described by comparing GC+CC vs GG. 
Table 4. Odds ratio for the associations of NBS1 c.8360G $>C$ polymorphism with clinicopathological features of breast cancer

\begin{tabular}{|c|c|c|}
\hline Genotype & $\begin{array}{l}\text { Odds ratio without adjustment for the age of breast } \\
\text { cancer diagnosis }(95 \% \text { confidence interval })^{\star}\end{array}$ & $\begin{array}{c}P \\
\text { value }\end{array}$ \\
\hline \multicolumn{3}{|l|}{ Odds of tumour size } \\
\hline NBS1 8360 GC vs $G G$ & $0.462(0.233-0.915)$ & 0.27 \\
\hline \multicolumn{3}{|l|}{ Odds of histological grade } \\
\hline NBS1 8360 GC versus $G G$ & $0.357(0.173-0.375)$ & 0.005 \\
\hline NBS1 8360 CC versus GG & $0.409(0.188-0.888)$ & 0.024 \\
\hline \multicolumn{3}{|l|}{ Odds of HER2 amplification } \\
\hline NBS1 8360 GC versus $G G$ & $0.226(0.099-0.513)$ & $<0.001$ \\
\hline NBS1 8360 CC versus $G G$ & $0.348(0.156-0.778)$ & 0.010 \\
\hline \multicolumn{3}{|c|}{ Odds of luminal B molecular BC type } \\
\hline NBS1 8360 GC versus $G G$ & $0.118(0.042-0.332)$ & $<0.001$ \\
\hline NBS1 8360 CC versus GG & $0.148(0.052-0.423)$ & $<0.001$ \\
\hline \multicolumn{3}{|c|}{ Odds of triple negative molecular BC } \\
\hline NBS1 8360 CC versus GG & $0.409(0.188-0.888)$ & 0.024 \\
\hline \multicolumn{3}{|l|}{ Odds of disease progress } \\
\hline NBS1 8360 GC versus GG & $0.462(0.233-0.915)$ & 0.027 \\
\hline NBS1 8360 CC versus GG & $0.240(0.098-0.585)$ & 0.002 \\
\hline
\end{tabular}

* Only statistically significant associations are shown.

0.156-0.778, $P=0.010)$ significantly increased the chances of HER2 amplification compared to $G G$ genotype. The data indicated that patients with 8360 GC genotype had a higher risk for breast cancer progression $(\mathrm{OR}=1.673,95 \% \mathrm{CI}$ $0.233-0.915, P=0.027)$. The carriers of homozygote 8360 CC had approximately six times higher risk for the disease progress $(\mathrm{OR}=5.946,95 \%$ CI $0.098-0.585, P=0.002$ ) when compared to $G G$ genotype. The prevalence of triple negative breast cancer subtype was significantly higher in individuals with NBS1 8360 CC genotype $(\mathrm{OR}=2.186,95 \%$ CI 0.188-0.888, $P=0.024)$. Regarding c.30537G $>C$, none of the genotypes had any significant influence on pathological characteristics.

\section{DISCUSSION}

Well-designed analysis of mutations or polymorphisms of the NBS1 gene or other DNA repair genes could potentially identify prognostic and predictive factors for breast cancer. c.657_661del mutation in the NBS1 gene clearly has association with an increased breast cancer risk, missense substitutions, e.g., p.I171 V and p.R215W, may represent cancer susceptibility alleles with lower penetrance (Bogdanova et al., 2008a).

Several studies have investigated not only the risk of breast cancer but also clinical characteristics of the disease in the case of NBS1 mutation. Previous report about the influence of NBS1 gene p.I171V mutation on histologi$\mathrm{cal}$ and clinical breast cancer features suggested accumulation of the negative prognostic factors (Roznowski et al., 2008). In the study by Roznowski and colleagues (2008), the carriers of p.I171 V showed a trend of a more aggressive cancer phenotype: higher histological grade, HER2 overexpression, lower oestrogen receptor expression, and familial history of malign disease. According to Nowak et al. (2008), NBS1 p.I171V mutation may be a general susceptibility factor to solid tumours. The study by Huzarski et al. 
(2013) showed that NBS1 c.657_661del did not affect breast cancer prognosis. In this case-control analysis, tumour size, grade, receptors, and lymph node status were also included. No statistically significant differences between mutation carriers and non-carriers were observed. Moreover, several studies have investigated the relation between NBS1 gene c.8360G $>C$ and c.30537G $>C$ polymorphisms and cancer risk, but the results were inconclusive (He et al., 2014). Sun et al. (2007) revealed that NBS1 c.8360G $>C$ polymorphism $C$ genotype may be one of the predictive neutropenic fever factors for breast cancer patients. All mentioned clinicopathological breast cancer characteristics and NBS1 gene status association analysis included case-control groups, but there is still a need of prospective studies with a larger sample size and with broader ethnic diversity.

In the present study, we investigated the possible association between mutations and polymorphisms in the NBS1 gene and clinicopathological characteristics of breast cancer. Compared with other studies (Roznowski et al., 2007; Huzarski et al., 2013; Rusak et al., 2019), our study is rather unique. We included young ( $\leq 50$ years of age) premenopausal women with I or II stage breast cancer diagnosis. The stage of breast cancer is a strong prognostic factor; in order to avoid its influence, we chose women with an early stage of breast cancer. To reveal the relation between NBS1 gene substitutions and clinicopathological characteristics, our analysis involved features that represent breast cancer phenotype and disease progress. No carriers of c.657_661del, p.I171V and p.R215W mutations were found. It might be due to the relatively young breast cancer patients' age, as it is known that mutations in the NBS1 gene more often influence malign disease in elderly patients. In the current study, some statistically significant associations were determined. NBS1 c.8360G $>C$ polymorphism $G C$ and $C C$ genotypes had effect on the histological tumour grade. It was observed that heterozygote $G C$ and homozygote $C C$ genotype holders had an increased risk for a higher histological grade. Furthermore, we revealed that $C C$ genotype of $c .8360 G>C$ in- creased the odds of HER 2 overexpression, which is a strong prognostic breast cancer factor. It was determined that current polymorphism heterozygous $G C$ and homozygous $C C$ genotypes increased the chances of breast cancer progression if compared with $G G$ genotype. According to our results, NBS1 c.8360G $>C$ polymorphism $C C$ genotype increased the odds of triple negative breast cancer. In the case of $c .30537 G>C$ polymorphism, no significant associations with breast cancer characteristics were found.

Unfortunately, there are some difficulties concerning the conclusion about the NBS1 gene status and breast cancer clinicopathological characteristics association because of small group of analysed patients compared with studies conducted by other researchers. We have to admit that the second limitation of our study is a short follow-up period of our patients.

\section{CONCLUSIONS}

Our data suggest that NBS1 gene c.8360G $>C$ polymorphism may be associated with breast cancer aggressiveness in young breast cancer patients. Further work is warranted to investigate the effect of the NBS1 status on breast cancer prognosis and malignancies risk.

\section{COMPETING INTEREST}

The authors declare that they have no competing interests.

\section{ACKNOWLEDGEMENTS}

We would like to thank the patients for their participation in this study.

Received 8 February 2021

Accepted 3 March 2021

\section{References}

1. Bogdanova N, Feshchenko S, Schürmann P, Waltes R, Wieland B, Hillemanns P, Rogov YI, Dammann O, Bremer M, Karstens JH, 
Sohn C, Varon R, Dörk T. Nijmegen Breakage Syndrome mutations and risk of breast cancer. Int J Cancer. 2008a; 122(4): 802-6.

2. Bogdanova N, Schürmann P, Waltes R, Feshchenko S, Zalutsky IV, Bremer M, Dörk T. NBS1 variant I171V and breast cancer risk. Breast Cancer Res Treat. 2008b; 112: 75-9.

3. Cybulski C, Górski B, Debniak T, Gliniewicz B, Mierzejewski M, Masojć B, Jakubowska A, Matyjasik J, Złowocka E, Sikorski A, Narod SA, Lubiński J. NBS1 is a prostate cancer susceptibility gene. Cancer Res. 2004; 64(4): 1215-9.

4. Cybulski C, Kluźniak W, Huzarski T, Wokołorczyk D, Kashyap A, Rusak B, et al. Polish Hereditary Breast Cancer Consortium. The spectrum of mutations predisposing to familial breast cancer in Poland. Int J Cancer. 2019; 145(12): 3311-20.

5. di Masi A, Antoccia A. NBS1 heterozygosity and cancer risk. Curr Genomics. 2008; 9(4): 275-81.

6. Ferlay J, Soerjomataram I, Ervik M, Dikshit R, Eser S, Mathers C, Rebelo M, Parkin DM, Forman D, Bray F. GLOBOCAN 2012 v1.1, Cancer Incidence and Mortality Worldwide: IARC CancerBase No. 11. France (Lyon): International Agency for Research on Cancer. 2012. Available from: https://publications. iarc.fr/Databases/Iarc-Cancerbases/GLOBOCAN-2012-Estimated-Cancer-IncidenceMortality-And-Prevalence-Worldwide-In2012-V1.0-2012.

7. He YZ, Chi XS, Zhang YC, Deng XB, Wang JR, Lv WY, Zhou YH, Wang ZQ. NBS1 Glu185Gln polymorphism and cancer risk: update on current evidence. Tumor Biol. 2014; 35(1): 675-87.

8. Huzarski T, Cybulski C, Jakubowska A, Byrski T, Gronwald J, Domagała P, et al. Clinical characteristics of breast cancer in patients with an NBS1 mutation. Breast Cancer Res Treat. 2013; 141(3): 471-6.

9. Lima ZS, Ghadamzadeh M, Arashloo FT, Amjad G, Ebadi MR, Younesi L. Recent advances of therapeutic targets based on the molecular signature in breast cancer: genetic mutations and implications for current treatment paradigms. J Hematol Oncol. 2019; 12(1): 38.

10. Lu J, Wei Q, Bondy ML, Li D, Brewster A, Shete S, Yu TK, Sahin A, Meric-Bernstam F, Hunt KK, Singletary SE, Ross MI, Wang LE. Polymorphisms and haplotypes of the NBS1 gene are associated with risk of sporadic breast cancer in non-Hispanic white women $\leq 55$ years. Carcinogenesis. 2006; 27(11): 2209-16.

11. Lu M, Lu J, Yang X, Yang M, Tan H, Yun B, Shi L. Association between the NBS1 E185Q polymorphism and cancer risk: a meta-analysis. BMC Cancer. 2009; 9: 124.

12. Nowak J, Mosor M, Ziółkowska I, Wierzbicka M, Pernak-Schwarz M, Przyborska M, Roznowski K, Pławski A, Słomski R, Januszkiewicz D. Heterozygous carriers of the I171V mutation of the NBS1 gene have a significantly increased risk of solid malignant tumors. Eur J Cancer. 2008; 44(4): 627-30.

13. Resnick IB, Kondratenko I, Pashanov E, Maschan AA, Karachunsky A, Togoev O, et al. 657 del5 mutation in the gene for Nijmegen breakage syndrome (NBS1) in a cohort of Russian children with lymphoid tissue malignancies and controls. Am J Med Genet. 2003; 120A(2): 174-9.

14. Roznowski K, Januszkiewicz-Lewandowska D, Mosor M, Pernak M, Litwiniuk M, Nowak J. I171V germline mutation in the NBS1 gene significantly increases risk of breast cancer. Breast Cancer Res Treat. 2008; 110: 343-8.

15. Rusak B, Kluźniak W, Wokołorczyk D, Stempa K, Kashyap A, Rudnicka $\mathrm{H}$, et al. Allelic modification of breast cancer risk in women with an NBN mutation. Breast Cancer Res Treat. 2019; 178(2): 427-31.

16. Steffen J, Varon R, Mosor M, Maneva G, Maurer $M$, Stumm $M$, et al. Increased cancer risk of heterozygotes with NBS1germline mutations in Poland. Int J Cancer. 2004; 111: 67-71.

17. Steffen J, Nowakowska D., Niwińska A, Czapczak D, Kluska A, Piątkowska M, Wiśniewska A 
Paszko Z. Germline mutations 657del5 of the NBS1 gene contribute significantly to the incidence of breast cancer in Central Poland. Int J Cancer. 2006; 119: 472-5.

18. Sun A, Yu T, Wang L, Lu J, Gonzales G, Pusztai L, Singletary S, Ross MI, Wei Q, Buchholz TA. Nijmegen breakage syndrome 1 (NBS1) gene polymorphism and chemotherapy-induced neutropenic fever in breast cancer patients. J Clin Oncol. 2007; 25(18S): 574.

19. Varon R, Seemanova E, Chrzanowska K, Hnateyko O, Piekutowska-Abramczuk D, Krajewska-Walasek M, Sykut-Cegielska J, Sperling K, Reis A. Clinical ascertainment of Nijmegen breakage syndrome (NBS) and prevalence of the major mutation, 657del5, in three Slav populations. Eur J Hum Genet. 2000; 8: 900-2.

20. Wang Z, Cui D, Lu W. NBS1 8360G > C polymorphism is associated with breast cancer risk: a meta-analysis. Breast Cancer Res Treat. 2010; 123(2): 557-61.

\section{Roberta Vadeikienè, Aistė Savukaityte், Rasa Ugenskienè, Jurgita Gudaitienè, Elona Juozaitytè}

\section{NBS1 GENO POKYČIŲ IR KLINIKINIŲ PATO- LOGINIŲ CHARAKTERISTIKŲ RYŠYS KRŪ- TIES VĖŽIU SERGANČIŲ JAUNŲ PACIENČIŲ GRUPE்JE}

\section{Santrauka}

Šios bandomosios studijos tikslas - nustatyti klinikinius požymius, kurie yra potencialiai susiję su NBS1 geno mutacijomis ir polimorfizmu krūties vèžiu sergančių jaunų ( $\leq 50$ metų) pacienčių grupejje. Kraujo èminiai paimti iš 80 pacienčių. Polimerazès grandininès reakcijos-restrikcijos fragmentų ilgio polimorfizmo metodu ištirta c. 657_661del, p. R215W ir p. $I 171 \mathrm{~V}$ mutacijos bei c. $8360 \mathrm{G}>\mathrm{C}$, c. $30537 \mathrm{G}>\mathrm{C}$ polimorfizmas. Statistinèje analizeje taikytas dvipusis Chi-kvadrato testas, o galimybių santykis ịvertintas pasitelkus logistinę regresiją. Tiriamojoje grupeje pacienčių su c. 657_661del, p. R215W ir p. $1171 \mathrm{~V}$ mutacijomis neaptikta. NBS1 c. $8360 G>C$ logistinè regresija rodo, kad GC ( $\breve{S} S=2,885,95 \%$ PI 0,173-0,735, $P=0,005)$ ir CC ( $\check{S} S=2,186,95 \%$ PI $0,188-0,888, P=0,024)$ genotipų nešiotojoms, palyginti su $G G$ genotipu, būdinga mažesnè blogai diferencijuoto naviko rizika. 8360 CC ( $\breve{S} S=3,034$, $95 \%$ PI 0,156-0,778, $P=0,010)$ genotipas reikšmingai padidino HER2 amplifikacijos galimybę, palyginti su GG genotipu. Progresuojančio krūties vėžio rizika reikšmingai didesnè NBS1 8360 GC $(\check{S} S=1,673,95 \%$ PI 0,233-0,915, $P=0,027)$ genotipo atveju. Homozigotinio 8360 CC genotipo nešiotojos turèjo beveik šešis kartus didesnę progresuojančios ligos riziką (ŠS $=5,946,95 \%$ PI 0,098-0,585, $P=0,002)$. Trigubai neigiamo krūties vėžio tipo atvejai reikšmingai dažniau pasitaikè asmenims su NBS1 8360 CC genotipu ( $̌ S ~=2,186$, $95 \%$ PI 0,188-0,888, $P=0,024)$. c. $30537 G>C$ polimorfizmo atveju reikšminga j̇taka patologinèms krūties vèžio charakteristikoms nenustatyta. NBS1 geno $c .8360 G>C$ polimorfizmas gali būti susijęs su krūties vèžio agresyvumu jaunų pacienčių grupèje.

Raktažodžiai: krūties vėžys, mutacija, polimorfizmas, NBS1 genas 